\title{
The Impact of Emotional Exhaustion on Psychological Factors in Workers with Secondary Traumatic Experiences: A Multi-Group Path Analysis
}

\author{
Min Jin Jin ${ }^{1,2 \star}$, Ji Sun Kim ${ }^{3 \star}$, Ho-Sung Lee ${ }^{4}$, Young Joon Kwon ${ }^{3}$, Se Hoon Shim ${ }^{3}$, Bum-Sung Choi ${ }^{5}$, \\ Dong-Woo Lee ${ }^{6}$, Jong-Woo Paik ${ }^{7}$, Boung Chul Lee ${ }^{8}$, Sung-Won Jung ${ }^{9}$, and Hwa-Young Lee ${ }^{3 凶}$ \\ ${ }^{1}$ Department of Psychiatry, Wonkwang University Hospital, Iksan, Republic of Korea \\ ${ }^{2}$ Institute of Liberal Education, Kongju National University, Gongju, Republic of Korea \\ ${ }^{3}$ Department of Psychiatry, Soonchunhyang University Cheonan Hospital, Cheonan, Republic of Korea \\ ${ }^{4}$ Department of Pulmonology and Allergy, Soonchunhyang University Cheonan Hospital, Cheonan, Republic of Korea \\ ${ }^{5}$ Department of Psychiatry, Medical Research Institute, Pusan National University Yangsan Hospital, Yangsan, Republic of Korea \\ ${ }^{6}$ Department of Psychiatry, Inje University Sanggye Paik Hospital, Seoul, Republic of Korea \\ ${ }^{7}$ Department of Psychiatry, Kyung Hee University School of Medicine, Seoul, Republic of Korea \\ ${ }^{8}$ Department of Neuropsychiatry, College of Medicine, Hallym University, Seoul, Republic of Korea \\ ${ }^{9}$ Department of Psychiatry, Keimyung University, School of Medicine, Daegu, Republic of Korea
}

Objective The objective of the present study was to explore causal pathways to understand how second traumatic experiences could affect the development of emotional exhaustion and psychiatric problems.

Methods A total of 582 workers who had jobs vulnerable to secondary traumatic experiences were enrolled for this study. Emotional exhaustion, secondary trauma, resilience, perceived stress, depression, anxiety, and sleep problems were evaluated. A model with pathways from secondary traumatic experience score to depression and anxiety was proposed. The participants were divided into three groups according to the resilience: the low, middle and high resilience group.

Results Resilience was a meaningful moderator between secondary traumatic experiences and psychiatric problems. In the path model, the secondary trauma and perceived stress directly and indirectly predicted perceived stress, emotional exhaustion, depression, anxiety, and sleep problems in all three groups. Direct effects of perceived stress on depression and anxiety were the largest in the low resilience group. However, direct effects of secondary trauma on perceived stress and emotional exhaustion were the largest in the high resilience group.

Conclusion Understanding the needs of focusing for distinct psychological factors offers a valuable direction for the development of intervention programs to prevent emotional exhaustion among workers with secondary traumatic experiences.

Psychiatry Investig 2020;17(11):1064-1072

Key Words Workers, Secondary traumatic experiences, Emotional exhaustion, Associated factors, Path analysis.

\section{INTRODUCTION}

Secondary traumatic stress has been defined as a natural

Received: December 4, 2019 Revised: January 31, 2020

Accepted: February 11, 2020

$\triangle$ Correspondence: Hwa-Young Lee, MD, PhD

Department of Psychiatry, Soonchunhyang University Cheonan Hospital, 31 Suncheonhyang 6-gil, Dongnam-gu, Cheonan 31151, Republic of Korea Tel: +82-41-570-3876, Fax: +82-41-592-3804, E-mail: leehy@schmc.ac.kr

*These authors contributed equally to this work.

(a) This is an Open Access article distributed under the terms of the Creative Commons Attribution Non-Commercial License (https://creativecommons.org/licenses/by$\mathrm{nc} / 4.0$ ) which permits unrestricted non-commercial use, distribution, and reproduction in any medium, provided the original work is properly cited. and consequential behavior and emotion resulting from knowing about the traumatizing event experienced by a significant other and the stress resulting from helping or wanting to help a traumatized or suffering person. ${ }^{1}$ A great deal of social work practice addresses client's crisis situations or helps clients deal with trauma that occurs in the aftermath of crisis. ${ }^{2}$ Providing trauma-intervention services places these workers at risk for traumatic responses themselves. ${ }^{3}$

Previous studies have used various synonyms of secondary traumatic stress such as burnout. ${ }^{4}$ It is best defined as a syndrome having symptoms of secondary traumatic stress and professional burnout. ${ }^{1,2,5,6}$ Professional burnout has been de- 
fined as a psychological state resulting from prolonged emotional or psychological stress on the job. ${ }^{7}$ Burnout could be defined as a psychological state resulting from emotional stress. The emotional exhaustion is a chronic state of physical and emotional depletion that results from excessive job and/or personal demands and continuous stress. ${ }^{8}$ Moreover, secondary traumatic experiences caused by repetitive demands for compassion could affect emotional exhaustion. ${ }^{9}$ Like secondary traumatic stress, reactions to burnout include emotional responses such as emotional exhaustion, ${ }^{10}$ depression, and anxiety. ${ }^{4}$

Emotional exhaustion is considered to have a high predictive value in anticipating the impact of stress on health in an active work population. ${ }^{7}$ Workers such as nurses, social workers, firefighters and police officers who have a job that is vulnerable to secondary traumatic experiences can also easily experience stress. ${ }^{211-14}$ Additionally, various studies have determined the relationship between depression and burnout. ${ }^{15}$ Although whether depression can be considered as a result of burnout or the opposite remains unclear and overlapping symptoms between twos have not been clearly solved yet either, ${ }^{15}$ the previous studies has found that burnout can develop into depression under certain conditions. ${ }^{16-18}$ Unfortunately, the vast majority of studies investigating this relationship is of crosssectional design; however, on the basis of results of previous studies, the causal models including secondary traumatic experiences, stresses, emotional exhaustion and symptoms of depression could be speculated. The secondary traumatic experiences could increase perceived stresses and stresses might affect emotional exhaustion of health professionals. Additionally, increased emotional exhaustion could affect the development of symptoms of depression.

Meanwhile, recent studies have shown a link between resilience and emotional exhaustion. ${ }^{19,20}$ Resilience appears to be protective against emotional exhaustion. ${ }^{21}$ Resilience is negatively associated with post-traumatic and depressive symptoms. ${ }^{22}$ Additionally, resilience can moderate the relationship between subjective experience about traumatic experiences and depressive symptoms. ${ }^{22}$

Despite above expected correlations between secondary traumatic experience-related factors and clinical importance, pathways to the development of psychiatric problems from secondary trauma have not been reported yet. By clarifying such pathways, the development of psychiatric problems from emotional exhaustion and perceived stress in workers with secondary traumatic experiences could be prevented.

Thus, the objective of the present study was to explore such pathways to understand how secondary traumatic experiences could affect the development of emotional exhaustion and psychiatric symptoms such as depression, anxiety and insom- nia. Additionally, this study aims to reveal the role of various psychological characteristics as risk and protective factors for the development of psychiatry problems. This study also evaluated whether resilience could moderate the development of psychiatric problems from secondary traumatic experiences.

\section{METHODS}

\section{Participants}

The study population initially included 615 participants and finally enrolled 582 participants (157 men and 425 women) who completed questionnaires with a mean age of $35.00 \pm 8.03$ years. The study population was enrolled from September 2015 to July 2017. This study included workers with secondary traumatic experience such as mental health worker, police officer, firefighter, paramedics, and nursing personnel working in a hospital's inpatient and outpatient units who were willing to participate in this study. The population had been working at workplaces that includes 6 cities in South Korea. This study and all protocols were approved by the Institutional Review Board and Ethics Committee of Soonchunhyang University Hospital (IRB number: 2015-05-013). It was performed in accordance with approved guidelines. Informed consent was obtained from all study participants.

\section{Psychological measures}

To assess emotional exhaustion, the Maslach Burnout Inventory-General Survey (MBI-GS) ${ }^{7,23}$ was administered. The MBI is the gold standard for detecting burnout. Furthermore, it has been shown to have adequate performance with a stable factorial structure among nurses from different populations in varied contexts. ${ }^{24}$ The MBI-GS, a 16-item questionnaire designed to assess the presence of burnout in healthcare providers, contains three subscales, each evaluating one of the underlying constructs of burnout: emotional exhaustion, depersonalization, and personal achievement. Each subscale comprises several items. Each item is rated by the respondent on a 7-point Likert scale according to the frequency of occurrence ( $0=$ "Never" to $6=$ "Every day"). The present study used emotional exhaustion, one subtype of MBI-GS, for the variable in the pathway model. There is a general consensus in the literature that emotional exhaustion is the central or core dimension of burnout. ${ }^{25,26}$

To measure the severity of depressive and anxiety symptoms, the Patient Health Questionnaire (PHQ) and Generalized Anxiety Disorder 7 (GAD-7) scale were used. ${ }^{27,28}$ To assess secondary traumatic stress, a secondary traumatic stress scale was administered. Secondary traumatic stress has been defined as "the natural, consequent behaviors and emotions resulting from knowledge about a traumatizing event experi- 
enced by a significant other." ${ }^{29}$ The scale consists of 17 items.

A 25-item Connor-Davidson Resilience Scale for measuring individual resilience in the face of stressful events was developed and evaluated. To assess individual stress, the 10-question Perceived Stress Scale (PSS) was applied. ${ }^{30}$ Quality of sleep was assessed with the Sleep Quality Scale. ${ }^{31}$ This scale has 28 items and six factors. ${ }^{31}$

\section{Statistical analysis}

To examine the moderating effect of resilience in the complex mediation model between the experience of secondary trauma and psychological symptoms, participants were divided into three groups: the low resilience group $(n=197)$, which was the lower $33.3 \%$ of participants with resilience scores of 6 to 54 , the middle resilience group $(n=199)$, which was the middle $33.4 \%$ of participants with resilience score of 55 to 69 , and the high resilience group $(n=186)$ which was the higher $33.3 \%$ of participants with resilience score of 70 to 100 .

Normality of variables by each group was tested before further analysis. Skewness over 2.0 and kurtosis over 7.0 were considered to reflect a moderately non-normal distribution. ${ }^{32}$
Correlation analyses were performed to examine relationships between secondary traumatic stress and psychological characteristics. All statistical analyses were conducted using SPSS 21.0 (IBM Corp., Armonk, NY, USA).

Based on correlation results, two models were hypothesized. One model is that secondary trauma can predict emotional exhaustion, which later predicts psychological symptoms. Another model is that secondary trauma can predict perceived stress, which then predicts emotional exhaustion and later predicts psychological symptoms.

Path analyses to determine a better-fit model were performed with the maximum likelihood estimator using AMOS 21.0. Chi-square test $\left(\chi^{2}\right)$, comparative fit index (CFI), normed fit index (NFI), Tucker-Lewis index (TLI), and root mean square error of approximation (RMSEA) indices were used to examine the goodness of fit of models. Cutoff points of indices were: CFI, NFI, TLI $>0.90$ and RMSEA $<0.08 .{ }^{33}$ For model comparison, a comparison with the Akaike Information Criterion (AIC) index was performed. The significance level was set at $\mathrm{p}<0.05$ (two-tailed).

Table 1. Comparison of baseline demographic, and psychological characteristics in participants with low, middle, and high resilience

\begin{tabular}{|c|c|c|c|c|c|c|}
\hline & $\begin{array}{l}\text { Low resilience }{ }^{\mathrm{a}} \\
\qquad(\mathrm{N}=197)\end{array}$ & $\begin{array}{l}\text { Middle resilience }{ }^{b} \\
\qquad(\mathrm{~N}=199)\end{array}$ & $\begin{array}{l}\text { High resilience }^{c} \\
\qquad(\mathrm{~N}=186)\end{array}$ & $\begin{array}{c}\text { Total } \\
(\mathrm{N}=582)\end{array}$ & \multirow[t]{2}{*}{$\mathrm{p}$} & \multirow{2}{*}{$\begin{array}{l}\text { Post-hoc test } \\
\text { using bonferroni }\end{array}$} \\
\hline & \multicolumn{4}{|c|}{ Mean \pm SD or $N(\%)$} & & \\
\hline Age (years) & $33.34 \pm 6.96$ & $34.44 \pm 7.74$ & $37.35 \pm 8.85$ & $35.00 \pm 8.03$ & $<0.001$ & $\mathrm{a}<\mathrm{c}, \mathrm{b}<\mathrm{c}$ \\
\hline Sex & & & & & $<0.001$ & \\
\hline Male & $34(17.3)$ & $50(25.1)$ & $73(39.2)$ & $157(26.3)$ & & \\
\hline Female & $163(82.7)$ & $149(74.9)$ & $113(60.8)$ & $425(71.1)$ & & \\
\hline Education (years) & $15.43 \pm 1.29$ & $15.61 \pm 1.36$ & $15.49 \pm 1.72$ & $15.51(1.46)$ & 0.45 & \\
\hline Marital status & & & & & 0.02 & \\
\hline Single & $137(69.5)$ & $120(60.3)$ & $96(51.6)$ & $353(60.7)$ & & \\
\hline Married & $55(27.9)$ & $78(39.2)$ & $86(46.2)$ & $219(37.6)$ & & \\
\hline Other & $5(2.6)$ & $1(0.5)$ & $4(2.2)$ & $10(1.7)$ & & \\
\hline Occupation status & & & & & 0.09 & \\
\hline Manager level & $17(8.6)$ & $26(13.1)$ & $30(16.1)$ & $73(12.5)$ & & \\
\hline Staff level & $180(91.4)$ & $173(86.9)$ & $156(83.9)$ & $509(87.5)$ & & \\
\hline Resilience scale & $46.29 \pm 8.76$ & $62.33 \pm 4.39$ & $80.87 \pm 10.03$ & $62.82 \pm 16.17$ & $<0.001$ & $\mathrm{a}<\mathrm{b}, \mathrm{a}<\mathrm{c}, \mathrm{b}<\mathrm{c}$ \\
\hline Secondary traumatic stress scale & $43.65 \pm 12.33$ & $35.83 \pm 11.04$ & $29.49 \pm 12.05$ & $36.45 \pm 13.13$ & $<0.001$ & $a>b, a>c, b>c$ \\
\hline Perceived stress scale & $19.46 \pm 4.91$ & $15.87 \pm 4.60$ & $11.88 \pm 6.14$ & $15.87 \pm 4.60$ & $<0.001$ & $a>b, a>c, b>c$ \\
\hline Maslach burnout inventory & & & & & & \\
\hline Emotional exhaustion & $25.73 \pm 6.00$ & $22.53 \pm 6.02$ & $18.18 \pm 7.86$ & $22.22 \pm 7.32$ & $<0.001$ & $\mathrm{a}>\mathrm{b}, \mathrm{a}>\mathrm{c}, \mathrm{b}>\mathrm{c}$ \\
\hline Depersonalization & $15.18 \pm 4.14$ & $12.50 \pm 4.21$ & $9.69 \pm 4.56$ & $12.51 \pm 4.84$ & $<0.001$ & $a>b, a>c, b>c$ \\
\hline Personal achievement & $25.63 \pm 4.60$ & $28.84 \pm 4.48$ & $33.39 \pm 5.02$ & $29.21 \pm 5.66$ & $<0.001$ & $\mathrm{a}<\mathrm{b}, \mathrm{a}<\mathrm{c}, \mathrm{b}<\mathrm{c}$ \\
\hline Depression scale & $8.92 \pm 6.08$ & $5.87 \pm 4.70$ & $4.25 \pm 4.90$ & $6.38 \pm 5.60$ & $<0.001$ & $a>b, a>c, b>c$ \\
\hline Anxiety scale & $5.44 \pm 4.65$ & $2.96 \pm 2.95$ & $2.17 \pm 3.08$ & $3.55 \pm 3.90$ & $<0.001$ & $a>b, a>c$ \\
\hline Sleep scale & $61.74 \pm 13.44$ & $56.08 \pm 12.76$ & $50.38 \pm 12.24$ & $56.18 \pm 13.62$ & $<0.001$ & $a>b, a>c, b>c$ \\
\hline
\end{tabular}




\section{RESULTS}

\section{Descriptive statistics and correlation analyses}

A total of 582 healthcare providers completed the survey. They had a mean age (standard deviation) of 35.0 (8.0) years. Descriptive and psychological characteristics of healthcare providers are shown in Table 1.

Correlation analyses among secondary traumatic stress, emotional exhaustion (subscale of $\mathrm{MBI}$ ), and other psychological measures were executed by each group since all scores of each group were within the range of normal distribution. As shown in Table 2, all correlation coefficients were significant.

Two models were hypothesized for the path analysis after correlation analyses. The first model was composed of a path from secondary trauma score to emotional exhaustion and paths between emotional exhaustion and other psychological symptoms based on previous studies. ${ }^{4,9}$ The second model was composed of an additional variable of perceived stress between secondary trauma and emotional exhaustion based on results of correlation analyses. These two hypothesized models are shown in Figure 1.

\section{Path analyses}

In order to reveal a better fitting model, the two hypothesized models were examined and compared with the AIC score of the pool of total participants. The AIC score of the first hypothesized model was 437.025 while that of the second hypothesized model was 173.278 . The second hypothesized model fit
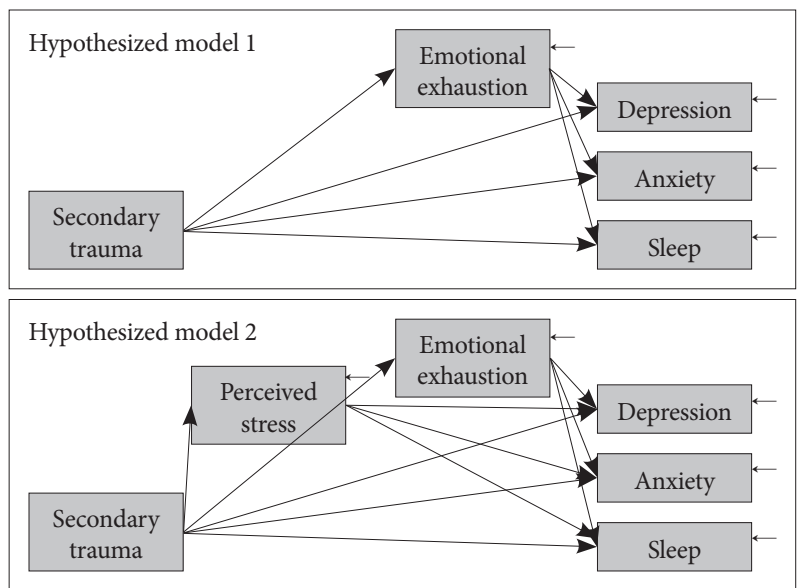

Figure 1. Two hypothesized models for path analysis.

Table 2. Correlations of psychological measures in participants with low, middle, and high resilience

\begin{tabular}{|c|c|c|c|c|c|c|}
\hline \multirow{2}{*}{ Measures } & \multicolumn{6}{|c|}{$\mathrm{r}$} \\
\hline & 1 & 2 & 3 & 4 & 5 & 6 \\
\hline \multicolumn{7}{|l|}{ Low resilience group $(\mathrm{N}=197)$} \\
\hline 1. Secondary trauma & - & & & & & \\
\hline 2. Perceived stress & $0.460^{* * *}$ & - & & & & \\
\hline 3. Emotional exhaustion & $0.406^{* * *}$ & $0.433^{* * *}$ & - & & & \\
\hline 4. Depression & $0.637^{* * *}$ & $0.590^{* * *}$ & $0.522^{* * *}$ & - & & \\
\hline 5. Anxiety & $0.637^{* * *}$ & $0.585^{* * *}$ & $0.402^{* * *}$ & $0.826^{* * *}$ & - & \\
\hline 6. Sleep & $0.588^{* * *}$ & $0.477^{* * *}$ & $0.508^{* * *}$ & $0.685^{* * *}$ & $0.68^{* *}$ & - \\
\hline \multicolumn{7}{|c|}{ Middle resilience group $(\mathrm{N}=199)$} \\
\hline 1. Secondary trauma & - & & & & & \\
\hline 2. Perceived stress & $0.586^{* * *}$ & - & & & & \\
\hline 3. Emotional exhaustion & $0.435^{* * *}$ & $0.393^{* * *}$ & - & & & \\
\hline 4. Depression & $0.595^{* * *}$ & $0.539^{* * *}$ & $0.484^{* * *}$ & - & & \\
\hline 5. Anxiety & $0.573^{* * *}$ & $0.554^{* * *}$ & $0.435^{* * *}$ & $0.729^{* * *}$ & - & \\
\hline 6. Sleep & $0.519^{* * *}$ & $0.361^{* * *}$ & $0.427^{* * *}$ & $0.721^{* * *}$ & $0.452^{* * *}$ & - \\
\hline \multicolumn{7}{|l|}{ High resilience group $(\mathrm{N}=186)$} \\
\hline 1. Secondary trauma & - & & & & & \\
\hline 2. Perceived stress & $0.708^{* * *}$ & - & & & & \\
\hline 3. Emotional exhaustion & $0.616^{* * *}$ & $0.614^{* * *}$ & - & & & \\
\hline 4. Depression & $0.724^{* * *}$ & $0.643^{* * *}$ & $0.670^{* * *}$ & - & & \\
\hline 5. Anxiety & $0.715^{* * *}$ & $0.644^{* * *}$ & $0.618^{* * *}$ & $0.829^{* * *}$ & - & \\
\hline 6. Sleep & $0.589^{* * *}$ & $0.435^{* * *}$ & $0.525^{* * *}$ & $0.682^{* * *}$ & $0.643^{* * *}$ & - \\
\hline
\end{tabular}

${ }^{* *} \mathrm{p}<0.01,{ }^{* * *} \mathrm{p}<0.001$ 
the data better than the first model since its AIC was much lower than that of the first model. Thus, the second hypothesized model was accepted and analyzed by groups. Moreover, additional correlation paths among depression, anxiety, and sleep problems were included based on modification indices and earlier results of correlation analyses.

As shown in Table 3, results of path analyses of the three groups all showed great fit. Figure 2 presents the model with significant paths and standardized parameter estimates of each group. However, results were slightly different from the results depending on group. The score of secondary trauma experi-

Table 3. Fitness indices of each model in participants with low, middle, and high resilience

\begin{tabular}{lccccccc}
\hline & $\chi^{2}$ & $\mathrm{df}$ & $\mathrm{p}$ & $\mathrm{CFI}$ & $\mathrm{NFI}$ & $\mathrm{TLI}$ & $\mathrm{RMSEA}$ \\
\hline $\begin{array}{l}\text { Low resilience } \\
\text { group }\end{array}$ & 1.422 & 1 & 0.233 & 0.999 & 0.998 & 0.990 & 0.046 \\
$\begin{array}{l}\text { Middle resilience } \\
\text { group }\end{array}$ & 0.229 & 1 & 0.632 & 1.000 & 1.000 & 1.022 & 0.000 \\
$\begin{array}{l}\text { High resilience } \\
\text { group }\end{array}$ & 0.547 & 1 & 0.460 & 1.000 & 0.999 & 1.009 & 0.000 \\
\hline
\end{tabular}

CFI: comparative fit index, NFI: normed fit index, TLI: TuckerLewis index, RMSEA: root mean square error of approximation ence significantly predicted perceived stress, emotional exhaustion, depression, anxiety, and sleep problems in all three groups.

In addition, the score of perceived stress significantly predicted emotional exhaustion, depression, anxiety, and sleep problems in all three groups. However, the path from emotional exhaustion to anxiety was only significant in the middle resilience group and the high resilience group $(\beta=0.179$, $\mathrm{p}=0.003 ; \beta=0.231, \mathrm{p}<0.001$, respectively), but not in the low resilience group ( $\beta=0.068, p=0.232$ ). On the other hand, the direct path from the perceived stress score to sleep problems was only significant in the low resilience group $(\beta=0.177$, $\mathrm{p}=0.004$ ), but not in the middle or the high resilience groups ( $\beta=0.035, p=0.632 ; \beta=-0.063, p=0.459$, respectively).

Direct effects of perceived stress on depression and anxiety were the largest in the low resilience group $(\beta=0.307, p<0.001$; $\beta=0.349, p<0.001$, respectively), but the smallest in the high resilient group $(\beta=0.148, p=0.030 ; \beta=0.194, p=0.006$, respectively). However, direct effects of secondary trauma on perceived stress and emotional exhaustion were the largest in the high resilience group $(\beta=0.708, p<0.001 ; \beta=0.365$, $p<0.001$, respectively), but the smallest in the low resilient group ( $\beta=0.460$,
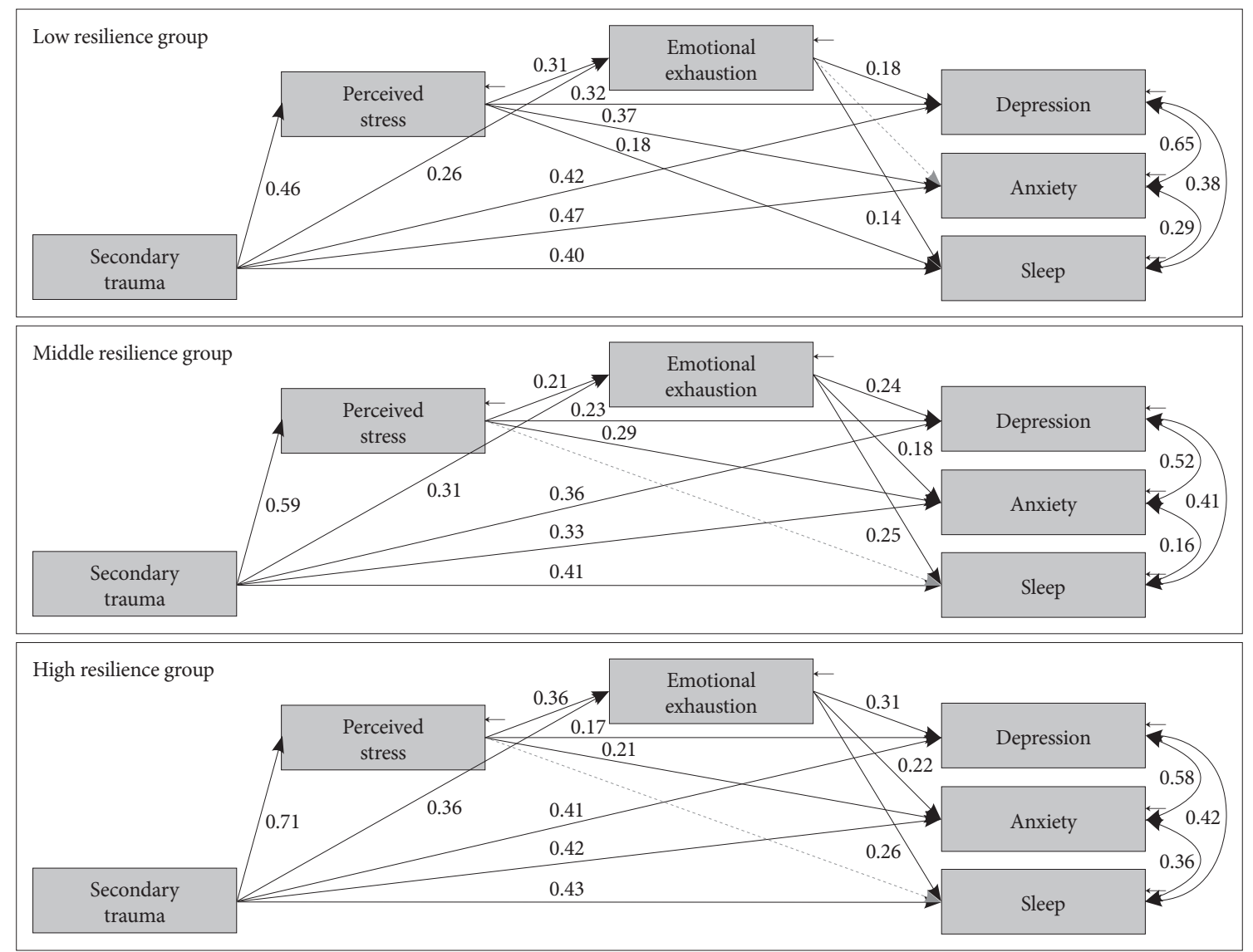

Figure 2. A comprehensive model from secondary traumatic experience to depression, anxiety, and sleep with mediators including perceived stress and emotional exhaustion in groups with low, middle, and high resilience. 
Table 4. Regression weight, standard error, and consistency ratio of models

\begin{tabular}{|c|c|c|c|c|c|c|c|c|c|}
\hline & \multicolumn{3}{|c|}{ Low resilience group } & \multicolumn{3}{|c|}{ Middle resilience group } & \multicolumn{3}{|c|}{ High resilience group } \\
\hline & $\begin{array}{c}\text { Standardized } \\
\text { regression } \\
\text { weights }\end{array}$ & SE & CR & $\begin{array}{l}\text { Standardized } \\
\text { regression } \\
\text { weights }\end{array}$ & SE & CR & $\begin{array}{l}\text { Standardized } \\
\text { regression } \\
\text { weights }\end{array}$ & SE & CR \\
\hline Secondary trauma $\rightarrow$ perceived stress & $0.460^{* * *}$ & 0.025 & 7.256 & $0.586^{* * *}$ & 0.024 & 10.188 & $0.708^{* * *}$ & 0.026 & 13.638 \\
\hline Secondary trauma $\rightarrow$ emotional exhaustion & $0.263^{* * *}$ & 0.034 & 3.751 & $0.311^{* * *}$ & 0.042 & 4.005 & $0.365^{* * *}$ & 0.051 & 4.697 \\
\hline Secondary trauma $\rightarrow$ depression & $0.405^{* * *}$ & 0.027 & 7.300 & $0.351^{* * *}$ & 0.029 & 5.202 & $0.423^{* * *}$ & 0.028 & 6.199 \\
\hline Secondary trauma $\rightarrow$ anxiety & $0.448^{* * *}$ & 0.022 & 7.743 & $0.322^{* * *}$ & 0.018 & 4.665 & $0.436^{* * *}$ & 0.018 & 6.096 \\
\hline Secondary trauma $\rightarrow$ sleep & $0.396^{* * *}$ & 0.067 & 6.439 & $0.393^{* * *}$ & 0.087 & 5.227 & $0.463^{* * *}$ & 0.087 & 5.384 \\
\hline Perceived stress $\rightarrow$ emotional exhaustion & $0.312^{* * *}$ & 0.086 & 4.460 & $0.211^{* *}$ & 0.101 & 2.721 & $0.355^{* * *}$ & 0.099 & 4.569 \\
\hline Perceived stress $\rightarrow$ depression & $0.307^{* * *}$ & 0.070 & 5.449 & $0.240^{* * *}$ & 0.067 & 3.628 & $0.148^{*}$ & 0.054 & 2.167 \\
\hline Perceived stress $\rightarrow$ anxiety & $0.349^{* * *}$ & 0.056 & 5.955 & $0.295^{* * *}$ & 0.043 & 4.357 & $0.194^{* *}$ & 0.036 & 2.724 \\
\hline Perceived stress $\rightarrow$ sleep & $0.177^{* *}$ & 0.171 & 2.846 & 0.035 & 0.204 & 0.479 & -0.063 & 0.171 & -0.740 \\
\hline Emotional exhaustion $\rightarrow$ depression & $0.224^{* * *}$ & 0.055 & 4.103 & $0.237^{* * *}$ & 0.046 & 3.992 & $0.319^{* * *}$ & 0.038 & 5.222 \\
\hline Emotional exhaustion $\rightarrow$ anxiety & 0.068 & 0.044 & 1.195 & $0.179^{* *}$ & 0.030 & 2.933 & $0.231^{* * *}$ & 0.025 & 3.610 \\
\hline Emotional exhaustion $\longrightarrow$ sleep & $0.270^{* * *}$ & 0.136 & 4.464 & $0.242^{* * *}$ & 0.140 & 3.651 & $0.278^{* * *}$ & 0.120 & 3.620 \\
\hline Depression error $\leftrightarrow$ anxiety error & $0.646^{* * *}$ & 1.107 & 7.597 & $0.520^{* * *}$ & 0.619 & 6.49 & $0.582^{* * *}$ & 0.513 & 6.842 \\
\hline Depression error $\leftrightarrow$ sleep error & $0.381^{* * *}$ & 3.054 & 4.979 & $0.412^{* * *}$ & 2.796 & 5.364 & $0.415^{* * *}$ & 2.300 & 5.219 \\
\hline Anxiety error $\leftrightarrow$ sleep error & $0.289^{* * *}$ & 2.373 & 3.885 & $0.163^{*}$ & 1.686 & 2.266 & $0.358^{* * *}$ & 1.481 & 4.582 \\
\hline
\end{tabular}

${ }^{*} \mathrm{p}<0.05,{ }^{* *} \mathrm{p}<0.01,{ }^{* * *} \mathrm{p}<0.001$. SE: standard error, CR: consistency ratio

$\mathrm{p}<0.001 ; \beta=0.263, \mathrm{p}<0.001$, respectively). Table 4 shows regression weights, standard errors, and consistency ratios of the model of each group.

\section{DISCUSSION}

In the present study, several psychological factors were associated with secondary traumatic experiences among workers who had a job vulnerable to secondary traumatic experiences. Moreover, secondary traumatic experiences significantly affected perceived stress, emotional exhaustion, depression, anxiety, and insomnia. Further, a model was developed including paths from secondary traumatic experiences to psychiatric problems such as depression, anxiety, and insomnia in workers both directly and indirectly through perceived stress and emotional exhaustion. More importantly, resilience moderated the path from perceived stress to emotional exhaustion and psychiatric problems.

In the present study, regardless of the degree of resilience, the model (path) showed that secondary traumatic experience could affect perceived stress, emotional exhaustion, and psychiatric problems such as depression, anxiety, and insomnia. Thus, a model was developed including the paths from secondary traumatic experiences to psychiatric problems such as depression, anxiety, and insomnia in workers both directly and indirectly through perceived stress and emotional exhaustion. This result is comparable to previous studies showing that secondary traumatic experiences could affect the development of depression in workers who help the population exposed to trauma. ${ }^{22,34,35}$ Anxiety and insomnia in our study were also affected by secondary trauma. This is expected as they are often comorbid symptoms of depression. ${ }^{36}$

Moreover, the pathway showed that emotional exhaustion, the core dimension of burnout, ${ }^{25,26}$ affected the development of depression and anxiety. Although various studies have reported the relation between depression and burnout, ${ }^{15,18}$ whether depression can be considered as a result of burnout or the opposite remains unclear. ${ }^{15}$ Several studies have found that burnout is a different concept from depression ${ }^{37,38}$ and that burnout can develop into depression. ${ }^{15,18,39}$ However, other studies have revealed that burnout and depression are associated with each other bidirectionally and that they have similar and overlapping concept. ${ }^{17,40}$ There is still confusion about the difference between burnout and depression ${ }^{15}$ because depression and emotional exhaustion have common features such as mood states. ${ }^{15,41}$ The present study contributes to prove the reasoning to distinguish depression from burnout. One study has shown that burnout can be used as a predictor of depressive symptoms in work life, ${ }^{15,41,42}$ especially work lives of healthcare providers who have a job that is vulnerable to secondary traumatic experiences.

Our study revealed that conditions such as depression and anxiety were related to greater emotional exhaustion. Similarly, emotional exhaustion was associated with greater health 
problems in the form of anxiety, depression, and insomnia. ${ }^{43}$ A previous structural equation modeling study by Glass et al. ${ }^{18}$ has concluded that a perceived lack of job control, in an expression similar to burnout, may indirectly drive depression. A literature review has noted that depression and burnout are distinct concepts. The causal relationship between them remains unclear. ${ }^{17}$ Although the present study showed that burnout could serve as a predictor of depressive symptoms in work life, ${ }^{15,39}$ other studies have concluded that depressive symptoms may predict emotional exhaustion. ${ }^{16}$ Further longitudinal studies are needed to clarify the causal relationship between depression and burnout.

This study disclosed the role of perceived stress as a mediator in the development of emotional exhaustion from secondary traumatic experiences. Regardless of the degree of resilience, the model (path) showed that perceived stress of individuals played a role in developing emotional exhaustion from secondary traumatic experience. According to conservation of resources theory, the general stress framework, the exposure to a broad range of stressors may deplete resources and lead to resource exhaustion. ${ }^{44}$ Stresses to work-related indirect exposure to traumatic events are examples of such broad range of stressors. This broad range of stressors may consequently lead to exhaustion of a broad range of resources. ${ }^{35}$ Emotional exhaustion may represent one of the facets of loss/exhaustion of resources. $^{35}$

Moreover, our results showed that secondary traumatic experiences could affect the development of emotional exhaustion. Cieslak et al. ${ }^{45}$ have indicated that there are strong associations between burnout and secondary traumatic stresses among human services professionals. Although there is no evidence clarifying whether secondary traumatic stresses can lead to burnout or burnout leads to secondary traumatic stresses, ${ }^{35}$ results of the present study were in line with our previous study on nursing personnel which showed that secondary traumatic experiences could predict emotional exhaustion in a regression analysis. ${ }^{16}$

Results of this study showed that resilience was negatively associated with various psychopathology such as depression, anxiety, burnout, and secondary traumatic stresses among workers who had a job vulnerable to secondary traumatic experiences. Additionally, the higher the resilience, the lower the direct effect from perceived stress to depression and anxiety in the population. The current findings add to a growing body of evidence on the healthy effect of resilience. ${ }^{46,47}$ Previous studies have suggested one potential explanation for the role of resilience is that it can affect the capacity of coping successfully with adversity. ${ }^{48}$ Higher resilience is usually associated with more positive emotion and increased emotional flexibility, which may reduce the likelihood of depression and post-trau- matic reactions..$^{22,49-51}$ Ying et al. ${ }^{22}$ have suggested that individual difference in resilience appears to be useful to understand the effective adaptation to extreme adversity. Additionally, the finding of this study is consistent with previous studies showing that dispositional resilience plays an important role in buffering the effect of the frequency of daily hassles on concurrent levels of symptoms distress. ${ }^{22,52}$ Findings of the current study suggest that resilience can serve as a "buffer" against perceptions of stress and psychopathology related to secondary traumatic experiences.

However, the higher the resilience, the lower the direct effect from secondary traumatic experiences to perceived stress and emotional exhaustion in the population. This suggests that individuals with higher resilience might be less influenced by secondary traumatic events. However, the impact of secondary trauma might be higher than that in those with lower trait resilience. While those individuals with higher levels of resilience did show a less significant increase in secondary traumatic stresses in the workplace, resilience might have a considerable impact on the development of burnouts and psychiatric problems. That is, individuals with higher resilience might have higher predictive powers of secondary traumatic experiences than those with lower levels of resilience. Therefore, not only understanding how one can develop and enhance resilience, but also coping and managing perceived stress response are of great importance to prevent the development of psychiatric illnesses such as depression and posttraumatic stress disorder.

This study has some limitations. First, there was a lack of information on several occupational covariates that might be associated with secondary traumatic experiences and burnout, such as types of secondary trauma or additional work performed or workload. Therefore, we could not include them in the analysis. Because the present study included various workers such as police officers, firefighters, social workers, and nurses to show various work conditions known to influence secondary traumatic experiences, this study could not apply one criterion on occupational status. Further studies that include additional explanatory variables related to secondary trauma and burnout with larger sample sizes are needed. Second, scales used in this study were self-report measures. However, these are reliable and validated instruments that have been widely used in other studies. Additionally, although we used path analysis to test predictive value, the present study was a crosssectional study. Further longitudinal studies are needed to clarify the causal relationship between secondary traumatic stress and psychopathologies. Finally, our results may not be generalizable to populations of other ethnicities.

Despite the above limitations, the present study also has some strengths. This is the first study to make and verify the possible candidate pathway from secondary traumatic stress 
to the development of emotional exhaustion and psychiatric problems in those with high-risk profession. Second, identification of protective and risk factors associated with psychiatric problems related to secondary traumatic stresses may help us identify groups at high risk of exhaustion and potentially modifiable risk factors that contribute to emotional exhaustion, a critical step for developing effective preventative strategies for this vulnerable population. For example, professions with less resilience and higher perceived stress could be supported by additional assistants or might receive stress management training to prevent burnout and depression. Third, this study used a relatively large sample size to achieve the study aims. Finally, the present study can help us determine whether interventions might be effective in preventing psychiatric problems in high-risk professions. Identifying the target of interventions could help professions who are at risk of exhaustion to choose proper psychological interventions.

In conclusion, our data showed that secondary traumatic experiences significantly affected perceived stress, emotional exhaustion, depression, anxiety, and insomnia. A model was developed including paths from secondary traumatic experiences to psychiatric problems such as depression, anxiety, and insomnia in workers both directly and indirectly through perceived stress and emotional exhaustion. Moreover, the path revealed that resilience could moderate the path from perceived stress to emotional exhaustion and psychiatric problems. Our results might offer insight into the necessities of beforehand interventions to prevent burnout and depression in high risk professions that are vulnerable to secondary traumatic experiences.

\section{Acknowledgments}

Our study was supported by the grants from the National Center for Mental Health Research \& Education, the Seoul National Hospital, Republic of Korea (HM15C1113) and Soonchunhyang University Research Fund.

\section{Conflicts of Interest}

The authors have no potential conflicts of interest to disclose.

\section{Author Contributions}

Conceptualization: Ji Sun Kim. Data curation: Min Jin Jin. Formal analysis: Min Jin Jin. Funding acquisition: Hwa-Young Lee. Investigation: HoSung Lee, Young Joon Kwon, Se Hoon Shim, Bum-Sung Choi, Dong-Woo Lee, Jong-Woo Paik, Boung Chul Lee, Sung-Won Jung. Methodology: Min Jin Jin. Project administaration: Ho-Sung Lee, Young Joon Kwon, Se Hoon Shim, Bum-Sung Choi, Dong-Woo Lee, Jong-Woo Paik, Boung Chul Lee, Sung-Won Jung. Resources: Hwa-Young Lee. Software: Ji Sun Kim. Supervision: Ho-Sung Lee, Young Joon Kwon, Se Hoon Shim, Bum-Sung Choi, Dong-Woo Lee, Jong-Woo Paik, Boung Chul Lee, Sung-Won Jung, HwaYoung Lee. Validation: Hwa-Young Lee. Visualization: Min Jin Jin. Writing -original draft: Min Jin Jin and Ji Sun Kim. Writing_-review \& editing: Hwa-Young Lee.

\section{ORCID iDs}

Min Jin Jin https://orcid.org/0000-0001-5494-5702
Ji Sun Kim https://orcid.org/0000-0003-2472-4591 Ho-Sung Lee https://orcid.org/0000-0001-6614-6465 Young Joon Kwon https://orcid.org/0000-0001-9340-0895 Se Hoon Shim https://orcid.org/0000-0002-3137-6591 Bum-Sung Choi https://orcid.org/0000-0002-1043-000X Dong-Woo Lee https://orcid.org/0000-0002-6383-1974 Jong-Woo Paik https://orcid.org/0000-0002-1804-8497 Boung Chul Lee https://orcid.org/0000-0002-0968-087X Sung-Won Jung https://orcid.org/0000-0002-2300-742X Hwa Young Lee https://orcid.org/0000-0002-2749-6232

\section{REFERENCES}

1. Figley CR. Compassion Fatigue as Secondary Traumatic Stress Disorder: An Overview. New York: Brunner/Mazel; 1995.

2. Newell JM, MacNeil GA. Professional burnout, vicarious trauma, secondary traumatic stress, and compassion fatigue: A review of theoretical terms, risk factors, and preventive methods for clinicians and researchers. Best Pract Ment Health Int J 2010;6:57-68.

3. Palm KM, Polusny MA, Follette VM. Vicarious traumatization: potential hazards and interventions for disaster and trauma workers. Prehosp Disaster Med 2004;19:73-78.

4. Salston M, Figley CR. Secondary traumatic stress effects of working with survivors of criminal victimization. J Trauma Stress 2003;16:167174.

5. Adams RE, Boscarino JA, Figley CR. Compassion fatigue and psychological distress among social workers: a validation study. Am J Orthopsychiatry 2006;76:103-108.

6. Bride BE, Radney M, Figley CR. Measuring compassion fatigue. Clin Soc Work J 2007;35:155-163.

7. Maslach C, Schaufeli WB, Leiter MP. Job burnout. Annu Rev Psychol 2001;52:397-422.

8. Wright TA, Cropanzano R. Emotional exhaustion as a predictor of job performance and voluntary turnover. J Appl Psychol 1998;83:486-493.

9. Sodeke-Gregson EA, Holttum S, Billings J. Compassion satisfaction, burnout, and secondary traumatic stress in UK therapists who work with adult trauma clients. Eur J Psychotraumatol 2013;4.

10. Prosser D, Johnson S, Kuipers E, Szmukler G, Bebbington P, Thornicroft G. Mental health, "burnout' and job satisfaction among hospital and community-based mental health staff. Br J Psychiatry 1996;169: 334-337.

11. Grant HB, Lavery CF, Decarlo J. An exploratory study of police officers: low compassion satisfaction and compassion fatigue. Front Psychol 2018;9:2793.

12. Greinacher A, Derezza-Greeven C, Herzog W, Nikendei C. Secondary traumatization in first responders: a systematic review. Eur J Psychotraumatol 2019;10:1562840.

13. Hall LH, Johnson J, Watt I, Tsipa A, O'Connor DB. Healthcare staff wellbeing, burnout, and patient safety: a systematic review. PLoS One 2016; 11:e0159015.

14. Salyers MP, Bonfils KA, Luther L, Firmin RL, White DA, Adams EL, et al. The relationship between professional burnout and quality and safety in healthcare: a meta-analysis. J Gen Intern Med 2017;32:475-482.

15. Papathanasiou IV. Work-related mental consequences: mplications of burnout on mental health status among health care roviders. Acta Inform Med 2015;23:22-28.

16. Choi BS, Kim JS, Lee DW, Paik JW, Lee BC, Lee JW, et al. Factors associated with emotional exhaustion in South Korean nurses: a crosssectional study. Psychiatry Investig 2018;15:670-676.

17. Glass D, McKnight J. Perceived control, depressive symptomatology, and professional burnout: A review of the evidence. Psychol Health 1996;11:23-48.

18. Glass DC, McKnight JD, Valdimarsdottir H. Depression, burnout, and perceptions of control in hospital nurses. J Consult Clin Psychol 1993; 61:147-155. 
19. Edward KL. The phenomenon of resilience in crisis care mental health clinicians. Int J Ment Health Nurs 2005;14:142-148.

20. Howard S, Johnson B. Resilient teachers: resisting stress and burnout. Soc Psychol Educ 2004;7:399-420.

21. Manzano G, Ayala-Calvo JC. Emotional exhaustion of nursing staff: influence of emotional annoyance and resilience. Int Nurs Rev 2012; 59:101-107.

22. Ying L, Wu X, Lin C, Jiang L. Traumatic severity and trait resilience as predictors of posttraumatic stress disorder and depressive symptoms among adolescent survivors of the Wenchuan earthquake. PLoS One 2014;9:e89401.

23. Shin KH. The Maslach Bunout Inventory-General Survey (MBI-GS): an application in South Korea. Korean J Indust Organ Psychol 2003; 16:1-17.

24. Poghosyan L, Aiken LH, Sloane DM. Factor structure of the Maslach burnout inventory: an analysis of data from large scale cross-sectional surveys of nurses from eight countries. Int J Nurs Stud 2009;46:894902.

25. Gaines J, Jermier J. Emotional exhaustion in a high stress organization. Acad Manag J 1983;26:567-586.

26. Sonnentag S, Kuttler I, Fritz C. On stressors, emotional exhaustion, and need for recovery: a multi-source study on the benefits of psychological detachment. J Vocation Behav 2010;76:355-365.

27. Spitzer RL, Kroenke K, Williams JB, Lowe B. A brief measure for assessing generalized anxiety disorder: the GAD-7. Arch Intern Med 2006;166:1092-1097.

28. Kroenke K, Spitzer RL, Williams JB. The PHQ-9: validity of a brief depression severity measure. J Gen Intern Med 2001;16:606-613.

29. Bride, BE, Robinson, MM, Yegidis B, Figley CR. Development and validation of the secondary traumatic stress scale. Res Soc Work Pract 2004;14:27-35.

30. Cohen S, Kamarck T, Mermelstein R. A global measure of perceived stress. J Health Soc Behav 1983;24:385-396.

31. Yi H, Shin K, Shin C. Development of the sleep quality scale. J Sleep Res 2006;15:309-316.

32. Curran PJ, West SG, Finch JF. The robustness of test statistics to nonnormality and specification error in confirmatory factor analysis. Psychol Methods 1996;1:16-29.

33. Kline RB. Principles and Practice of Structural Equation Modeling. 3rd Edition. New York: The Guilford Press; 2011.

34. Gilroy PJ, Carroll L, Murra J. A preliminary survey of counseling psychologists' personal experiences with depression and treatment. Prof Psychol Res Pract 2002;33:402-407.

35. Shoji K, Lesnierowska M, Smoktunowicz E, Bock J, Luszczynska A, Benight CC, et al. What comes first, job burnout or secondary traumatic stress? Findings from two longitudinal studies from the U.S. and
Poland. PLoS One 2015;10:e0136730.

36. Buysse DJ, Angst J, Gamma A, Ajdacic V, Eich D Rossler W. Prevalence, course, and comorbidity of insomnia and depression in young adults. Sleep 2008;31:473-480.

37. Demerouti E, Bakker AB, Nachreiner F, Schaufeli WB. The job demands-resources model of burnout. J Appl Psychol 2001;86:499-512.

38. Iacovides A, Fountoulakis KN, Moysidou C, Ierodiakonou C. Burnout in nursing staff: is there a relationship between depression and burnout? Int J Psychiatry Med 1999;29:421-433.

39. Duquette A, Kerouac S, Sandhu BK, Beaudet L. Factors related to nursing burnout: a review of empirical knowledge. Issues Ment Health Nurs 1994; 15:337-358.

40. Iacovides A, Fountoulakis KN, Kaprinis S, Kaprinis G. The relationship between job stress, burnout and clinical depression. J Affect Disord 2003;75:209-221.

41. Tanaka JS, Huba GJ. Confirmatory hierarchical factor analysis of psychological distress measures. J Pers Soc Psychol 1984;46:621-635.

42. Firth H, McKeown P, McIntee J, Britton P. Professional depression, 'burnout' and personality in longstay nursing. Int J Nurs Stud 1987;24: 227-237.

43. van Servellen G, Topf M, Leake B. Personality hardiness, work-related stress, and health in hospital nurses. Hosp Top 1994;72:34-39.

44. Hobfoll SE. Conservation of resources. A new attempt at conceptualizing stress. Am Psychol 1989;44:513-524.

45. Cieslak R, Shoji K, Douglas A, Melville E, Luszczynska A, Benight CC. A meta-analysis of the relationship between job burnout and secondary traumatic stress among workers with indirect exposure to trauma. Psychol Serv 2014;11:75-86.

46. Bonanno GA. Clarifying and extending the construct of adult resilience. Am Psychol 2005;60:265-267.

47. Ong AD, Zautra AJ, Reid MC. Psychological resilience predicts decreases in pain catastrophizing through positive emotions. Psychol Aging 2010;25:516-523.

48. Block J, Kremen AM. IQ and ego-resiliency: conceptual and empirical connections and separateness. J Pers Soc Psychol 1996;70:349-361.

49. Ong AD, Bergeman CS, Bisconti TL, Wallace KA. Psychological resilience, positive emotions, and successful adaptation to stress in later life. J Pers Soc Psychol 2006;91:730-749.

50. Tugade MM, Fredrickson BL. Resilient individuals use positive emotions to bounce back from negative emotional experiences. J Pers Soc Psychol 2004;86:320-333.

51. Waugh CE, Thompson RJ, Gotlib IH. Flexible emotional responsiveness in trait resilience. Emotion 2011;11:1059-1067.

52. Pinquart M. Moderating effects of dispositional resilience on associations between hassles and psychological distress. J Appl Dev Psychol 2009;30:53-60. 